\title{
$L$-SUBALGEBRAS OF $M(G)\left({ }^{1}\right)$
}

\author{
BY \\ JOSEPH L. TAYLOR
}

Let $G$ be a locally compact abelian group with dual group $\Gamma$. Let $M(G)$ be the algebra of bounded regular Borel measures on $G$ under convolution multiplication, and let $L(G)$ be the subalgebra of $M(G)$ consisting of all absolutely continuous measures in $M(G)$. By an $L$-subalgebra of $M(G)$ we mean a closed subalgebra $N$, with the property that if $\mu \in N$ and $\nu \in M(G)$, with $\nu$ absolutely continuous with respect to $\mu$, then $\nu \in N$. Clearly $L(G)$ is an $L$-subalgebra of $M(G)$.

Let $N$ be an $L$-subalgebra of $M(G)$. If $\gamma \in \Gamma$ and $h_{\gamma}(\mu)=\int \bar{\gamma} d \mu$ for $\mu \in N$, then $h_{\gamma}$ is a multiplicative linear functional on $N$. Hence, if $\Delta$ is the space of all multiplicative linear functionals on $N$, then $\gamma \rightarrow h_{\gamma}$ maps $\Gamma$ into $\Delta$. If this map is one to one and onto, then we shall say that the maximal ideal space of $N$ is $\Gamma$.

Note that the maximal ideal space of $L(G)$ is $\Gamma$ (cf. [5, Chapter 1]), but $L(G)$ is not unique in this respect. If we define $(L(G))^{1 / 2}$ to be the intersection of all maximal ideals of $M(G)$ containing $L(G)$, then $(L(G))^{1 / 2}$ is also an $L$-subalgebra of $M(G)$ with maximal ideal space $\Gamma$ (cf. [7, Lemma 1 and Theorem 1]). If $\mu \in M(G)$ and $\mu^{n} \in L(G)$ for some $n$, then $\mu \in(L(G))^{1 / 2}$. Hewitt and Zuckerman have recently shown in [3], that for every nondiscrete 1.c.a. group $G$, there is a singular measure $\mu \in M(G)$ such that $\mu^{2} \in L(G)$. This shows that $L(G) \neq(L(G))^{1 / 2}$ if $G$ is nondiscrete.

Our main theorem (Theorem 1) characterizes completely those $L$-subalgebras of $M(G)$ that have maximal ideal space $\Gamma$. This result was conjectured in [7] and proved there for a special case. The missing ingredient for a proof in the general case is supplied by one of the results of [8].

THEOREM 1. If $N$ is an L-subalgebra of $M(G)$, then the maximal ideal space of $N$ is $\Gamma$ if and only if $L(G) \subset N \subset(L(G))^{1 / 2}$.

Proof. That $L(G) \subset N \subset(L(G))^{1 / 2}$ implies that $\Gamma$ is the maximal ideal space of $N$, is proved in [7].

According to Lemma 4 of [7], to prove the converse it is sufficient to show that if the maximal ideal space of $N$ is $\Gamma$, then $L(G) \cap N \neq(0)$. It follows from the proof of Theorem 2 of [7], that this problem can be reduced to the case where $G=R^{n} \times H$ with $H$ compact. If $n=0$ or 1, Lemma 5 and Theorem 2 of [7] show how to construct a nonzero measure in $N \cap L(G)$. The existence of such a measure for general $n$, is just the corollary to Theorem 6.2 of [8].

Presented to the Society, in part, January 24, 1967 under the title $L$-subalgebras of $M(G)$; received by the editors August 23, 1967.

( ${ }^{1}$ Research partially sponsored by the Air Force Office of Scientific Research, Office of Aerospace Research, United States Air Force, under AFOSR grant No. 1313-67. 
Of course, the key to the proof of Theorem 1 is the result that $N \cap L(G)$ contains a nonzero measure if the maximal ideal space of $N$ is $\Gamma$ (Theorem 6.2 of [8]). The proof of this result is somewhat involved. For those who do not wish to beat through [8], we present, in $\S 1$ of this paper, an outline of the process by which the above result is obtained in the case $G=R^{n}$.

In $\$ 2$ we study the implications of Theorem 1 for the structure theory of $M(G)$ and other convolution measure algebras.

1. Constructing a smooth measure in $N$. In this section, we assume that $G=R^{n}$ and $N$ is an $L$-subalgebra of $M(G)$ with maximal ideal space $\Gamma=R^{n}$. We set $N_{e}=\{\mu+z e: \mu \in N, z \in C\}$, where $e$ is the point mass at zero in $G$. The algebra $N_{e}$ is an $L$-subalgebra of $M(G)$ with maximal ideal space $\Gamma^{\prime}=\Gamma \cup\{\infty\}$, the onepoint compactification of $\Gamma=R^{n}$.

Definition 1.1. (a) If $A$ is a compact convex subset of $R^{n}$, we set

$$
\varphi_{A}(y)=\sup _{x \in A} e^{-x \cdot y}
$$

(b) if $\mu$ is a measure on $R^{n}$, we set $\|\mu\|_{A}=\int \varphi_{A}(y) d|\mu|(y)$;

(c) we denote by $\mathfrak{R}_{e}(A)$ the collection of all measures $\mu$ on $R^{n}$ for which $\|\mu\|_{A}<\infty$ and the restriction of $\mu$ to each compact set is in $N_{e}$.

Note that if $\mu \in \mathfrak{R}_{e}(A)$, then the Laplace transform $\mu^{\wedge}(z)=\int e^{-z \cdot x} d \mu(x)$. exists for $z \in C^{n}$ with $\operatorname{Re} z \in A$.

In $\S 2$ of [8] we show that for each compact convex set $A \subset R^{n}$, the space $\mathfrak{N}_{e}(A)$ is a Banach algebra with identity under the norm \|\|$_{A}$ and convolution multiplication. If $\Phi \neq A \subset B$, then the identity map is a norm decreasing isomorphism of $\mathfrak{N}_{e}(B)$ into $\mathfrak{N}_{e}(A)$. Theorem 6.1 of [8] implies:

Lemma 1.1. The maximal ideal space of $\mathfrak{R}_{e}(A)$ is $\Delta(A)=\left\{z \in C^{n}: \operatorname{Re} z \in A\right\} \cup\{\infty\}$, where the Gelfand transform of $\mu \in \mathfrak{N}_{e}(A)$ coincides with the natural extension of the Laplace transform, $\mu^{\wedge}(z)=\int e^{-z \cdot x} d \mu(x)$, to $\Delta(A)$.

Let $A$ be an $n$-simplex in $R^{n}$, and let $A_{0}, A_{1}, \ldots, A_{n}$ be the $(n-1)$-faces of $A$. Let $\mu_{1}, \ldots, \mu_{n} \in \mathfrak{R}_{e}(A)$ be measures such that $\mu_{1}, \ldots, \mu_{n}$ do not vanish simultaneously at $\infty$ or on $\left\{z \in C^{n}: \operatorname{Re} z \in \partial A\right\}$. Consider the equation

$$
\mu_{1} \nu_{1}+\cdots+\mu_{n} \nu_{n}=e .
$$

Since the functions $\mu_{1}, \ldots, \hat{\mu_{n}}$ do not vanish simultaneously on

$$
\Delta\left(A_{i}\right)=\left\{z \in C^{n}: \operatorname{Re} z \in A_{i}\right\} \cup\{\infty\},
$$

it follows from Lemma 1.1 that $\left(^{*}\right)$ has a solution in $\mathfrak{N}_{e}\left(A_{i}\right)$ for each $i$. Hence, we can choose measures $\nu_{i j}(i=0, \ldots, n ; j=1, \ldots, n)$ such that $\mu_{1} \nu_{i 1}+\cdots+\mu_{n} \nu_{i n}=e$ for $i=0,1, \ldots, n$, and $\nu_{i j} \in \mathfrak{N}_{e}\left(A_{i}\right)$. Let $\rho_{k}$ be the determinant of the matrix obtained from $\left(\nu_{i j}\right)$ by deleting the $k$ th row. Note that $\rho_{k} \in \mathfrak{R}_{e}\left(A_{0} \cap \cdots \cap \hat{A}_{k} \cap \cdots \cap A_{n}\right)$, and $A_{0} \cap \cdots \cap \hat{A}_{k} \cap \cdots \cap A_{n}$ is the vertex of $A$ opposite the face $A_{k}$. We set 
$\rho=\sum_{k=0}^{n}(-1)^{k} \rho_{k}$. Note that $\rho$ is a (possibly nonfinite) measure such that the restriction of $\rho$ to each compact set is in $N_{e}$; i.e., $\rho$ is locally in $N_{e}$.

We call $\rho$ the residue measure for the equation $\left({ }^{*}\right)$ on the simplex $A$. The significance of this measure is indicated by the following lemma, which restates a portion of Theorem 4.2 of [8]:

LEMma 1.2. The equation $\left(^{*}\right)$ has a solution in $\mathfrak{N}_{e}(A)$ if and only if $\rho=0$.

In $\S 5$ of [8] we use the inverse Laplace transform and analytic function theory to prove the following:

LEMMA 1.3. The measure $\rho$ is absolutely continuous. In fact, its Radon-Nikodym derivative $h_{\rho}$ has the form $h_{\rho}(y)=\sum_{k=1}^{m} P_{k}(y) e^{z_{k} \cdot y}$ for polynomials $P_{1}, \ldots, P_{m}$ and $n$-tuples $z_{1}, \ldots, z_{m} \in \boldsymbol{C}^{n}$.

To prove that $N \cap L(G) \neq(0)$, it now suffices to choose $\mu_{1}, \ldots, \mu_{n} \in N_{e}$ such that $\mu_{1}, \ldots, \mu_{n}$ have compact support, $\mu_{1}(\infty) \neq 0$, and $\hat{\mu_{1}}, \ldots, \hat{\mu_{n}}$ have a nonempty discrete set of common zeros. In fact, we may then choose a simplex $A$ such "that $\left\{z \in C^{n}: \operatorname{Re} z \in A\right\}$ contains a common zero of $\hat{\mu_{1}}, \ldots, \hat{\mu_{n}}$ but $\left\{z \in C^{n}: \operatorname{Re} z \in \partial A\right\} \cup\{\infty\}$ does not. The residue measure $\rho$ for equation $\left(^{*}\right)$ on $A$ is nonzero, by Lemma 1.2 , and absolutely continuous, by Lemma 1.3. Since $\rho$ is locally in $N_{e}$, it follows that $N_{e} \cap L(G) \neq(0)$.

2. Applications of Theorem 1. In this section we shall use the terminology and concepts introduced in [10]. In [10] we defined the concept of convolution measure algebra. In particular, any $L$-subalgebra of $M(G)$ is a convolution measure algebra. If $M$ is a semisimple convolution measure algebra, then there exists a compact topological semigroup $S$ and an order preserving isomorphism-isometry $\mu \rightarrow \mu_{S}$ of $M$ into $M(S)$, such that

(1) the image of $M$ in $M(S)$ is weak-* dense;

(2) each multiplicative linear functional $h$ on $M$ has the form $h(\mu)=\int f d \mu_{S}$ for some semicharacter $f$ on $S$; and

(3) there are enough nonzero semicharacters on $S$ to separate points (cf. [10, §2]).

We call $S$ the structure semigroup of $M$. The space of nonzero semicharacters on $S$ is denoted by $S^{\wedge}$. Conditions (1), (2), and (3) characterize the structure semigroup $S$ and the embedding $\mu \rightarrow \mu_{S}$ to within equivalence. We may consider $S^{\wedge}$ to be the maximal ideal space of $M$ if we define the Gelfand transform $\mu \rightarrow \mu^{\wedge}$ by $\mu^{\wedge}(f)$ $=\int f d \mu_{S}$ for $f \in S^{\wedge}$ and $\mu \in M$, and give $S^{\wedge}$ the weakest topology under which all of the functions $\mu^{\wedge}$ for $\mu \in M$ are continuous. We call this topology the $\left(S^{\wedge}, M\right)$ topology.

If $p$ is an idempotent of $S$, let $K_{p}$ denote the maximal group of $S$ containing $p$. Let $K$ denote the union of all maximal groups of $S$. Note that $K$ is a compact sub-semigroup of $S$ and each of the groups $K_{p}$ is a compact topological group. 
Definition 2.1. (a) If $p=p^{2} \in S$ let $N_{p}=\left\{\mu \in M: \mu_{S}\right.$ is concentrated on $\left.K_{p}\right\}$, and set $M_{1}=\sum_{p=p^{2} \in S} \oplus N_{p}$.

(b) Let $M_{2}=\left\{\mu \in M: \mu_{S}\right.$ is concentrated on $K$ but $\left|\mu_{S}\right|\left(K_{p}\right)=0$ for each $\left.p\right\}$.

(c) Let $M_{3}=\left\{\mu \in M: \mu_{S}\right.$ is concentrated on $\left.S \backslash K\right\}$.

Each of the spaces $M_{1}, M_{2}$, and $M_{3}$ is an $L$-subspace of $M$, and $M=M_{1} \oplus M_{2}$ $\oplus M_{3}$. Also, $M_{1}$ and $M_{2}$ are $L$-subalgebras of $M$. This decomposition of $M$ illustrates that there are three basically different kinds of measures in a convolution measure algebra. Theorem 1 allows us to completely describe the nature of measures that lie in the space $M_{1}$. We do this in Theorem 3. The nature of the measures in $M_{2}$ and $M_{3}$ is less well understood. In Lemma 2.2 and 2.3 we give some information concerning these spaces which can easily be inferred from the definitions. Combining these results in the case $M=M(G)$, we obtain an existence theorem (Theorem 4) for "strange" multiplicative linear functionals on $M(G)$.

By an embedding of one convolution measure algebra in another, we mean an isomorphism which is order and norm preserving and has an $L$-subalgebra as its range.

THEOREM 2. Let $N$ be a semisimple convolution measure algebra with structure semigroup $S$. We have that $S$ is a group if and only if there is an l.c.a. group $G$ and an embedding $\mu \rightarrow \mu_{G}$, of $N$ as an L-subalgebra $N_{G}$ of $M(G)$, such that $L(G) \subset N_{G}$. $\subset(L(G))^{1 / 2}$. In this case, $S$ is the Bohr compactification of $G, S^{\wedge}$ with the $\left(S^{\wedge}, N\right)$ topology is the dual group of $G$, and the map $\mu \rightarrow \mu_{S}$ is the map $\mu \rightarrow \mu_{G}$ followed by the natural embedding of $M(G)$ in $M(S)$.

Proof. Note that if $S$ is a group, then each element of $S^{\wedge}$ is a character of $S$, and $S^{\wedge}$ is also a group. In fact, with the $\left(S^{\wedge}, N\right)$ topology, $S^{\wedge}$ is an 1.c.a. group. Let $G$ be its dual group. It follows that $S$ is the Bohr compactification of $G$, since $S$ is compact and has dual group $S^{\wedge}$ (cf. [5, Chapter 1]).

For each $\mu \in N$, the function $\mu^{\wedge}(f)=\int f d \mu_{S}$ is continuous on $S^{\wedge}$ in the $\left(S^{\wedge}, N\right)$ topology. Furthermore, if $\mu \geqq 0$ then $\mu_{S} \geqq 0$ and so $\mu^{\wedge}$ is a positive definite function on the group $S^{\wedge}$. It follows from 1.4 .3 of [5] that for each $\mu \in N$, there exists $\mu_{G} \in M(G)$ such that $\mu^{\wedge}(f)=\int_{S} f d \mu_{S}=\int_{G} f d \mu_{G}=\mu_{G}(f)$, where we consider $G$ as embedded in $S$. The map $\mu \rightarrow \mu_{G}$ embeds $N$ as a subalgebra $N_{G}$ of $M(G)$, and the map $\mu \rightarrow \mu_{S}$ is just $\mu \rightarrow \mu_{G}$ followed by the natural embedding of $M(G)$ in $M(S)$. Since $N_{S}=\left\{\mu_{S}: \mu \in N\right\}$ is an $L$-subalgebra of $M(S)$ (cf. Theorem 2.3 of [10]), it follows that $N_{G}$ is an $L$-subalgebra of $M(G)$.

Note that the maximal ideal space of $N_{G}$ is the dual group $\Gamma$ of $G$, since $\Gamma$ is just $S^{\wedge}$ with the $\left(S^{\wedge}, N\right)$ topology. Hence, Theorem 1 implies that

$$
L(G) \subset N_{G} \subset(L(G))^{1 / 2} .
$$

Conversely, if $N$ can be embedded as an $L$-subalgebra $N_{G}$ of $M(G)$ for some $G$, and $L(G) \subset N_{G} \subset(L(G))^{1 / 2}$, then Theorem 1 implies that the dual group $\Gamma$ of $G$ 
is the maximal ideal space of $N$. It follows that the Bohr compactification of $G$ is the structure semigroup for $N$. This completes the proof.

Recall from $\S 1$ of [10] that the notation $\mu \perp \nu$ means $\mu$ and $\nu$ are mutually singular. If $N$ is an $L$-subspace of a convolution measure algebra $M$, then $N^{\perp}=\{\mu \in M: \mu \perp \nu$ for every $\nu \in N\}$, and $M=N \oplus N^{\perp}$.

Let $M$ be a semisimple convolution measure algebra with structure semigroup $S$, and let $K_{p}$ and $N_{p}$ be as in Definition 2.1.

LEMMA 2.1. If $p$ is an idempotent of $S$ for which $N_{p} \neq(0)$, then

(a) there is an open-compact prime ideal $J_{p} \subset S$ such that $K_{p}$ is the kernel (minimal ideal) of $S \backslash J_{p}$;

(b) if $M_{p}=\left\{\mu \in M: \mu_{S}\right.$ is concentrated on $\left.S \backslash J_{p}\right\}$, then $N_{p}$ is an ideal of $M_{p}$ and $M_{p}^{\perp}$ is an ideal of $M$.

(c) The structure semigroup of $N_{p}$ is $K_{p}$.

Proof. If $N_{p} \neq(0)$ then we can choose a nonnegative measure $\mu \in N_{p}$ with $\|\mu\|=1$. Let $A_{p}=\left\{f \in S^{\wedge}: \mu^{\wedge}(f)=1\right\}$. Clearly $A_{p}$ is compact in the $\left(S^{\wedge}, M\right)$ topology. Furthermore, $f \in A_{p}$ if and only if $f \in S^{\wedge}$ and $f \equiv 1$ on carrier $\left(\mu_{S}\right)$. It follows that $A_{p}$ is closed under pointwise multiplication. Pointwise multiplication is a separately continuous operation on $S^{\wedge}$ in the $\left(S^{\wedge}, M\right)$ topology (cf. [10, §3]). Thus, $A_{p}$ is a compact topological semigroup with a separately continuous operation. This is enough to imply the existence of the kernel for $A_{p}$ and an idempotent $\pi$ in this kernel (cf. [4, Theorem 2.3]). If $f \in A_{p}$ then $f$ is in the kernel of $A_{p}$ if and only if $|f|=\pi$. It follows that for $f \in A_{p},|f| \pi=|f \pi|=\pi$.

If $J_{p}=\{s \in S: \pi(s)=0\}$, then $J_{p}$ is an open-compact prime ideal of $S$ (cf. [10, Theorem 3.2]). Since $\mu$ is concentrated on $K_{p}$ and $\mu^{\wedge}(\pi)=1$, it follows that $K_{p} \subset S \backslash J_{p}$. Let $q$ be the idempotent in the kernel of $S \backslash J_{p}$. Since $p \in K_{p} \subset S \backslash J_{p}$, we have $p q=q$. If $p \neq q$ then there exists $f \in S^{\wedge}$ such that $f(p) \neq f(q)$. This implies that $f(p)=1$ and $f(q)=0$. However, if $f(p)=1$ then $|f| \equiv 1$ on $K_{p}$ and $|f| \in A_{p}$. Hence, $|f| \pi=\pi$. This is impossible since $\pi(q)=1$. Hence, $p=q$ and $K_{p}$ is the kernel of $S \backslash J_{p}$. This completes the proof of Part (a).

Part (b) follows immediately from Part (a). In fact, if $\mu \in N_{p}$ and $\nu \in M_{p}$, then $\mu_{S}$ is concentrated on $K_{p}, \nu_{S}$ is concentrated on $S \backslash J_{p}$, and $\mu_{S} \cdot \nu_{S}=(\mu \cdot \nu)_{S}$ is concentrated on $K_{p}$ since $K_{p}$ is an ideal of $S \backslash J_{p}$. Thus, $\mu \cdot \nu \in N_{p}$, and $N_{p}$ is an ideal of $M_{p}$. Clearly, $M_{p}^{\perp}=\left\{\mu \in M: \mu_{S}\right.$ is concentrated on $\left.J_{p}\right\}$. It follows that $M_{p}^{\perp}$ is an ideal of $M$.

To prove that $K_{p}$ is the structure semigroup of $N_{p}$, we must verify that conditions (1), (2), and (3), listed at the beginning of this section, are satisfied. Since $S^{\wedge}$ separates points in $S$, the restrictions of elements of $S^{\wedge}$ to $K_{p}$ separate points in $K_{p}$. Hence (3) is satisfied. Let $h$ be a multiplicative linear functional on $N_{p}$. We can extend $h$ to a m.l.f. $h^{\prime}$ on $M_{p}$ by setting $h^{\prime}(\nu)=h(\mu \cdot \nu) / h(\mu)$ for some $\mu \in N_{p}$ with $h(\mu) \neq 0$. If $\nu \in M$ we can write $\nu$ uniquely in the form $\rho+\omega$, where $\rho \in M_{p}$ and $\omega \in M_{p}^{\perp}$. We set $h^{\prime \prime}(\nu)=h^{\prime}(\rho)$. It follows that $h^{\prime \prime}$ is a m.l.f. on $M$ which agrees with 
$h$ on $N_{p}$. Since $S$ is the structure semigroup of $M$, there exists $f \in S^{\wedge}$ such that $h^{\prime \prime}(\nu)=\int f d \nu_{S}$ for every $\nu \in M$. The restriction of $f$ to $K_{p}$ is an element of $K_{p}^{\wedge}$ which yields the m.l.f. $h$ on $N_{p}$. Hence, condition (2) is satisfied.

To complete the proof of (c), we must show that (1) is satisfied, i.e., that $\left(N_{p}\right)_{S}=\left\{\mu_{S}: \mu \in N_{p}\right\}$ is weak-* dense in $M\left(K_{p}\right)$. We have that $M_{S}$ is weak-* dense in $M(S)$. Since $S \backslash J_{p}$ is both open and closed, it follows that $\left(M_{p}\right)_{S}$ is weak-* dense in $M\left(S \backslash J_{p}\right)$. Let $K_{p}^{\prime}$ be the smallest closed subset of $K_{p}$ on which every element of $\left(N_{p}\right)_{S}$ is concentrated. We must show that $K_{p}^{\prime}=K_{p}$. Note that $K_{p}^{\prime}$ is a compact semigroup, since $N_{p}$ is an algebra. It follows that $K_{p}^{\prime}$ is a compact subgroup of $K_{p}$. If $A=\left\{s \in S: s K_{p}^{\prime} \subset K_{p}^{\prime}\right\}$, then $A$ is a compact subset of $S \backslash J_{p}$. If $K_{p}^{\prime} \neq K_{p}$ then $A \neq S \backslash J_{p}$. However, since $N_{p}$ is an ideal of $M_{p}$ and every element of $\left(N_{p}\right)_{S}$ is concentrated on $K_{p}^{\prime}$ it follows that every element of $\left(M_{p}\right)_{S}$ is concentrated on $A$. Hence $A=S \backslash J_{p}$ and $K_{p}^{\prime}=K_{p}$. This completes the proof.

In combination with Theorem 2, the above result yields the following:

THEOREM 3. Let $M$ be a semisimple convolution measure algebra with structure semigroup $S$. Let $p$ be an idempotent of $S$ and let $K_{p}$ and $N_{p}$ be as in Definition 2.1. If $N_{p} \neq(0)$, there is an l.c.a. group $G_{p}$, having $K_{p}$ as Bohr compactification, such that $N_{p}$ can be embedded in $M\left(G_{p}\right)$ so that $L\left(G_{p}\right) \subset N_{p} \subset\left(L\left(G_{p}\right)\right)^{1 / 2}$.

Theorem 3 gives a fairly good picture of the space $M_{1}=\sum_{p=p^{2} \in S} \oplus N_{p}$. We do not have satisfactory descriptions of the spaces $M_{2}$ and $M_{3}$, however. The characterizations given in the next two lemmas are almost restatements of the definitions of $M_{2}$ and $M_{3}$.

Let $f$ be a semicharacter in $S^{\wedge}$. If $\mu \in M$, then there is a function $f_{\mu} \in L^{\infty}(\mu)$ such that $f(\nu)=\int f d \nu_{S}=\int f_{\mu} d \nu$ for every measure $\nu$ which is absolutely continuous with respect to $\mu$. If $M=M(G)$ then, since $f$ determines a multiplicative linear functional on $M,\left\{f_{\mu}\right\}_{\mu \in M}$ is a generalized character in the terminology of Sreìder in [6]. The properties of $f_{\mu}$ as a function in $L^{\infty}(\mu)$ are determined by the properties of $f$ as a continuous function on carrier $\left(\mu_{S}\right) \subset S$. In fact, it follows from Theorem 1.2 of [10], that the map $f \rightarrow f_{\mu}$ preserves pointwise multiplication and conjugation. Furthermore, $\left\|f_{\mu}\right\|_{\infty}=\sup \left\{|f(s)|: s \in \operatorname{carrier}\left(\mu_{S}\right)\right\}$.

LEMMA 2.2. If $\mu \in M$ then $\mu \in M_{2}$ if and only if

(1) for every $f \in S^{\wedge},\left|f_{\mu}\right|^{2}=\left|f_{\mu}\right|$; and

(2) if $E$ is any $\mu$-measurable set which is not a set of $\mu$-measure zero, then there exists $f \in S^{\wedge}$ such that $\left\{x \in E:\left|f_{\mu}(x)\right|=1\right\}$ and $\left\{x \in E:\left|f_{\mu}(x)\right|=0\right\}$ are both sets of nonzero $\mu$-measure.

Proof. By Lemma 3.4 of [10], $s \in K$ if and only if $|f(s)|^{2}=|f(s)|$ for every $f \in S^{\wedge}$. It follows that condition (1) of the lemma is equivalent to $\mu$ being concentrated on $K$.

Similarly, if $s, t \in K$ then $s$ and $t$ are in different maximal groups if and only if there exists $f \in S^{\wedge}$ such that $f$ is one at one of these points and zero at the other. 
It follows that condition (2) of the lemma is equivalent to the statement that no measure absolutely continuous with respect to $\mu$ is concentrated on a single maximal group. This, in turn, is equivalent to $\left|\mu_{S}\right|\left(K_{p}\right)=0$ for each $p$. The proof is complete, in view of the definition of $M_{2}$ (Definition 2.1).

Lemma 2.3. If $\mu \in M$ then $\mu \in M_{3}$ if and only if given any $\mu$-measurable set $E$, not of $\mu$-measure zero, there exists $f \in S^{\wedge}$ such that $\left\{x \in E: 0<\left|f_{\mu}(x)\right|<1\right\}$ has nonzero $\mu$-measure.

Proof. This follows immediately if we note that, for $s \in S, s \in S \backslash K$ if and only if there exists $f \in S^{\wedge}$ such that $0<|f(s)|<1$.

If $f \in S^{\wedge}, f \geqq 0$, and $\mu \in M$, then the function $z \rightarrow \mu^{\wedge}\left(f^{z}\right)=\int f^{z} d \mu_{S}=\int\left(f_{\mu}\right)^{z} d \mu$ is analytic for $\operatorname{Re} z>0$ (cf. [10, §3]). This function is nonconstant provided $\left\{x: 0<f_{\mu}(x)<1\right\}$ has nonzero $\mu$-measure. Thus, Lemma 2.3 illustrates that, for a measure $\mu$ in $M, \mu^{\wedge}$ behaves like a nonconstant analytic function on a portion of $S^{\wedge}$ provided $\mu$ has a nonzero projection on $M_{3}$.

We now interpret the preceding results in the case where $M=M(G)$ for some 1.c.a. group $G$.

LEMMA 2.4. Let $S$ be the structure semigroup of $M(G)$ and let $p$ be an idempotent of $S$. If $N_{p} \neq(0)$ then there exists an 1.c.a. group $G_{p}$ and a continuous isomorphism $\beta$ of $G_{p}$ onto $G$, such that $N_{p}=\bar{\beta}\left(L\left(G_{p}\right)\right)^{1 / 2}$, where $\bar{\beta} \mu(E)=\mu\left(\beta^{-1}(E)\right)$ for $\mu \in\left(L\left(G_{p}\right)\right)^{1 / 2}$.

Proof. By Theorem 3, we may embed $L\left(G_{p}\right)$ in $N_{p}$ through an isomorphism $\bar{\beta}$ which is order and norm preserving. Furthermore, $\bar{\beta} L\left(G_{p}\right)$ is an $L$-subalgebra of $N_{p}$ and, hence, of $M(G)$.

Recall from Lemma 2.1 that $N_{p}$ is an ideal of an $L$-subalgebra $M_{p}$, with $M_{p}^{\perp}$ an ideal of $M(G)$. It follows that $M_{p}$ must contain all discrete measures in $M(G)$. From this it follows that $N_{p}$ is a translation invariant subspace of $M(G)$. However, $\bar{\beta} L\left(G_{p}\right)$ is an ideal of $N_{p}$ and so $\bar{\beta} L\left(G_{p}\right)$ is also translation invariant. However, any nonzero translation invariant $L$-subspace of $M(G)$ is weak-* dense in $M(G)$. Hence, $\beta L\left(G_{p}\right)$ is weak-* dense in $M(G)$.

By the results of $\S 4.6$ of [5], the map $\bar{\beta}$ has an extension to an isomorphism $\bar{\beta}: M\left(G_{p}\right) \rightarrow M(G)$ which is also norm and order preserving. It follows that there is a continuous isomorphism $\beta: G_{p} \rightarrow G$ such that $\bar{\beta} \mu(E)=\mu\left(\beta^{-1}(E)\right)$ for $\mu \in M\left(G_{p}\right)$. Since $\bar{\beta} L\left(G_{p}\right)$ is translation invariant in $M(G)$, we have that $\beta$ is onto.

By Theorem 3, we have that $\bar{\beta} L\left(G_{p}\right) \subset N_{p} \subset \bar{\beta}\left(L\left(G_{p}\right)\right)^{1 / 2}$. However, recall that $N_{p}=\left\{\mu \in M(G): \mu_{S}\right.$ is concentrated on $\left.K_{p}\right\}$. It follows from Theorem 3 and [7, Lemma 1], that if $\mu \in \bar{\beta}\left(L\left(G_{p}\right)\right)^{1 / 2}$, then $\mu_{S}$ is concentrated on $K_{p}$ and, hence, $\mu \in N_{p}$. Thus, $N_{p}=\bar{\beta}\left(L\left(G_{p}\right)\right)^{1 / 2}$. This completes the proof.

Since the map $\beta: G_{p} \rightarrow G$, of the above lemma, is a continuous isomorphism onto, the group $G_{p}$ differs from the group $G$ only in that it possess a possibly stronger topology. Let $\mathscr{T}$ be any topology on $G$ such that $(G, \mathscr{T})$ is an 1.c.a. group and the identity map $i:(G, \mathscr{T}) \rightarrow G$ is continuous. Note that $M(G, \mathscr{T})$ and 
$L(G, \mathscr{T})$ may be considered $L$-subalgebras of $M(G)$. Also, the space $M(G, \mathscr{T})^{\perp}$ consists of all measures in $M(G)$ which are zero on every $\mathscr{T}$-compact set in $G$, and is an ideal of $M(G)$. Note that $L(G, \mathscr{T})$ is an ideal of $M(G, \mathscr{T})$. Let $(L(G, \mathscr{T}))^{1 / 2}$ denote the intersection of all maximal ideals of $M(G, \mathscr{T})$ containing $L(G, \mathscr{T})$. In view of Lemma 2.4, the decomposition given in Lemma 2.1 (b) may be described as follows: Let $p$ be an idempotent of $S$ for which $N_{p} \neq(0)$. There exists a topology $\mathscr{T}$ on $G$, of the above type, such that $M_{p}=M(G, \mathscr{T}), N_{p}=(L(G, \mathscr{T}))^{1 / 2}$, and $M_{p}^{\perp}=M(G, \mathscr{T})^{\perp}=\{\mu \in M(G): \mu(A)=0$ for every $\mathscr{T}$-compact set $A\}$.

A great deal of work has gone into the problem of constructing "strange" multiplicative linear functionals on $M(G)$; i.e., multiplicative linear functionals which behave differently than group characters (cf. [1], [2], [6], and [9]). These results usually involve constructing special kinds of measures on special kinds of subsets of $G$. Our final theorem is a general existence theorem for "strange" multiplicative linear functionals on $M(G)$.

THEOREM 4. Let $M_{1}(G)=\sum \mathscr{T}(L(G, \mathscr{T}))^{1 / 2}$, where $\mathscr{T}$ ranges over all topologies for which $(G, \mathscr{T})$ is an l.c.a. group and $i:(G, \mathscr{T}) \rightarrow G$ is continuous. If $\mu \in M(G)$ and $\mu \perp M_{1}(G)$, then, for each Borel set $E \subset G$ with $|\mu|(E) \neq 0$, there exists a multiplicative linear functional $f$ on $M(G)$ for which

$$
\left\{g \in E:\left|f_{\mu}(g)\right|>0\right\} \text { and }\left\{g \in E:\left|f_{\mu}(g)\right|<1\right\}
$$

both have nonzero $|\mu|$-measure.

Proof. This follows directly from Lemmas 2.2, 2.3, and 2.4 and the fact that $M(G)=M_{1} \oplus M_{2} \oplus M_{3}$.

Let $\mathscr{T}$ be a topology of the above type on $G$. By Lemma 1 of [7], we have $\mu \perp(L(G, \mathscr{T}))^{1 / 2}$ if and only if $\mu^{n} \perp L(G, \mathscr{T})$ for every $n$. Hence, the conclusion of Theorem 4 holds provided $\mu^{n} \perp L(G, \mathscr{T})$ for every $n$ and every $\mathscr{T}$.

Note that for many groups $G$, there are very few topologies $\mathscr{T}$ on $G$ of the type described in Theorem 4 . For $G=R$ there are only two-the ordinary topology and the discrete topology. Thus, we might interpret Theorem 4 as indicating that "strange" multiplicative linear functionals are the rule rather than the exception.

For a study of varying topologies on a group $G$, we refer the reader to [11].

We close this discussion by posing a question.

Question: Are there 1.c.a. groups $G$ for which $M_{2}(G) \neq(0)$ ? (Cf. Definition 2.1.)

It follows from the work of Hewitt and Kakutani in [1], that $M_{3}(G) \neq(0)$ for every nondiscrete group $G$.

\section{REFERENCES}

1. E. Hewitt and S. Kakutani, A class of multiplicative linear functionals on the measure algebra of a locally compact abelian group, Illinois J. Math. 4 (1960), 553-574.

2. - Some multiplicative linear functionals on M(G), Ann. of Math. 79 (1964), 489-505.

3. E. Hewitt and H. Zuckerman, Singular measures with absolutely continuous convolution squares, Proc. Cambridge Philos. Soc. 62 (1966), 339-420. 
4. K. de Leeuw and I. Glicksberg, Applications of almost periodic compactifications, Acta Math. 105 (1961), 63-97.

5. W. Rudin, Fourier analysis on groups, Interscience, New York, 1962.

6. Y. Sreider, The structure of maximal ideals in rings of measures with convolution, Amer. Math. Soc. Transl. (1) 8 (1962), 365-391.

7. J. L. Taylor, Convolution measure algebras with group maximal ideal spaces, Trans. Amer. Math. Soc. 128 (1967), 257-263.

8. - Ideals and Laplace transforms for a class of measure algebras on a group, Acta Math. (to appear).

9. - The Shilov boundary of the algebra of measures on a group, Proc. Amer. Math. Soc. 16 (1965), 941-945.

10. - The structure of convolution measure algebras, Trans. Amer. Math. Soc. 119 (1965), 150-166.

11. N. Th. Varopoulos, Studies in harmonic analysis, Proc. Cambridge Philos. Soc. 60 (1964), 465-516.

\section{UNIVERSITY OF UTAH}

Salt Lake City, Utah 